\title{
核酸功能化稀土基纳米材料在生物检测中的应用
}

\author{
贾伊祎 王文杰 ${ }^{\dagger}$ 梁玲袁荃* \\ (湖南大学 化学化工学院 化学生物学与纳米医学研究所 长沙 410082)
}

\begin{abstract}
摘要 体内一些生物分子和离子的水平通常与细胞、组织、器官等结构和功能的变化相关, 从而直接影响到疾病的预 防、诊断和治疗, 因此对体内这些物质的生物检测在医疗和健康领域具有重要的意义. 基于稀土基纳米材料构建的纳 米苂光探针具有灵敏度高、简单高效、抗干扰能力强等优点, 在生物检测方面具有巨大的潜力. 对稀土基纳米材料的 核酸功能化能够进一步为纳米荧光探针提供更好的特异性识别能力和生物相容性, 从而增强其在复杂样品中的生物检 测能力. 本综述总结了核酸功能化的稀土基纳米材料作为纳米苂光探针在生物检测领域的研究进展, 简要介绍了其主 要种类和性能、检测机理及检测物质, 最后对该领域面临的挑战及未来的发展方向进行了展望.
\end{abstract}

关键词 生物分子; 生物检测; 苂光探针; 纳米材料; 稀土元素; 核酸适配体

\section{Bioassay Applications of Aptamer-Functionalized Rare Earth Nanomaterials}

\author{
Jia, Yiyi ${ }^{\dagger} \quad$ Wang, Wenjie ${ }^{\dagger} \quad$ Liang, Ling Yuan, Quan* \\ (Institute of Chemical Biology and Nanomedicine, College of Chemistry and Chemical Engineering, Hunan University, \\ Changsha 410082, China)
}

\begin{abstract}
The levels of some biomolecules and ions in the body are usually related to the structural and functional changes of cells, tissues, organs, etc., which directly affect the prevention, diagnosis, and treatment of diseases. Therefore, in vivo bioassays of these substances are of great significance in medical and healthcare fields. The nano fluorescent probes consisted of rare earth nano materials have advantages of high sensitivity, simplicity, efficiency, and strong anti-interference ability, thus showing great potential in bioassays. The functionalization of aptamers on rare earth nanomaterials can further provide better specific recognition ability and biocompatibility for nano fluorescent probes, thereby enhancing their bioassays ability in complex samples. In this paper, the research progress of aptamer-functionalized rare earth nanomaterials as nano fluorescent probes in the field of bioassays is reviewed, and the main types, properties, detection mechanisms and detection substances are briefly introduced.
\end{abstract}

Keywords biomolecule; bioassay; fluorescent probe; nanomaterial; rare earth; aptamer

\section{1 引言}

疾病诊断和健康监测与我们的健康生活密不可分, 对体内一些生物分子或离子进行检测通常能够为病理 或生理过程提供客观准确的评价，从而反映人体的健康 状况. 比如在尿常规分析中, 尿液中维生素 C 含量的长 期增高可能与肾结石相关; 通过咽拭子标本采集和核酸 序列检测, 可以判断病人是否感染新冠病毒. 因此, 在 临床医学的应用中, 我们需要提升与疾病健康相关物质 的生物检测能力, 从而实现疾病的早期准确诊断以及生 理状态的监控. 纳米荧光探针是近几十年被提出的一类 能实现高效生物检测的光学传感器, 通常包含信号报告 单元、识别单元及连接基团等三个部分, 可以对生物分 子及离子产生快速灵敏的光学响应. 纳米荧光探针的信
号报告单元即荧光发射基团, 通常为具有高荧光效率的 有机苂光染料和具有发光性能的苂光纳米材料 ${ }^{[1]}$, 利用 荧光发射基团的发光变化可以对物质含量进行定性和 定量分析. 苂光发射基团的发光性能对纳米苂光探针的 检测灵敏度和准确性有重要影响, 开发合适的苂光发射 基团也因此成为了设计纳米荧光探针的重点. 稀土基纳 米材料是以稀土元素作为主要成分或掺杂剂的发光纳 米材料 ${ }^{[2]}$, 具有发射线尖锐、斯托克斯位移/反斯托克斯 位移大、苂光寿命长、量子产率高等优点 ${ }^{[3]}$, 被认为是 极具潜力的纳米苂光探针的发射基团. 其优异的光学性 能主要来源于稀土元素 $4 \mathrm{f}$ 电子层特殊的能级结构 ${ }^{[3 b, 3 c]}$. 因此稀土基发光纳米材料作为纳米苂光探针的发射基 团，可以有效避免光谱串扰和组织自发荧光干扰，极大

\footnotetext{
* E-mail: yuanquan@whu.edu.cn

$\dagger$ These authors contributed equally to this work

Received June 19, 2020; published July 27, 2020.

Project supported by the National Natural Science Foundation of China (No. 21904037), Natural Science Foundation of Hunan Province, China (Nos. 2020JJ4173, 2020JJ5038) and Changsha Municipal Science and Technology Projects, China (No. Kq1901030).

项目受国家自然科学基金(No. 21904037)、湖南省自然科学基金(Nos. 2020JJ4173, 2020JJ5038)和长沙市科技计划项目(No. Kq1901030)资助.
} 
地增强纳米荧光探针的稳定性和灵敏度, 提升疾病诊断 和健康监测的及时性和准确性.

除了发光性能优异的荧光发射基团外，纳米苂光探 针还需要连接基团充当枢纽连接识别待测物质的识别 单元, 来构建一个完整的具有定位识别能力的纳米苂光 探针. 识别单元能够特异性识别分析物并通过配位作用 或者氢键等与其结合, 常见的识别元件有抗体 ${ }^{[4]}$ 、核酸 适配体 ${ }^{[5]}$ 、抗生物素蛋白/生物素对 ${ }^{[6]}$ 和多肽 ${ }^{[7]}$ 等. 其中, 核酸适配体(aptamer)是指利用体外篮选一指数富集的 配体系统进化技术(SELEX)从特定的寡核苷酸文库中笁 选出的能与靶标物特异性结合的寡核苷酸序列一一脱氧 核糖核酸(DNA)或核糖核酸(RNA). 在 1990 年, Tuerk 与 Gold $^{[8]}$ 发明了 SELEX 技术, 并成功地笁选出了能够与 T4 DNA 聚合酶特异性结合的 RNA 寡核苷酸. 同年, Ellington 和 Szostak ${ }^{[9]}$ 也基于 SELEX 技术成功笁选出了 能够与汽巴克隆蓝、活性蓝 4 发生特异性结合的 RNA, 并提出了 “核酸适配体” 的概念. 核酸适配体作为纳米 荧光探针的识别元件, 与目标物以互补碱基配对、静电 作用或氢键等方式结合, 并且在结合的过程中, 为了与 目标物形状更加匹配, 其构型会折叠成稳定的三维空间 结构, 因此具有可与抗体媲美的高选择性和高亲和力. 其次, 核酸适配体的生物相容性好、性质稳定, 对纳米 材料的功能化能够降低纳米材料的毒性同时还能增强 纳米材料的血液循环能力 ${ }^{[10]}$. 最重要的是, 核酸适配体 的结合目标广, 可以用于多种目标物的测定, 包括金属 离子 ${ }^{[11]}$ 、小分子(如三磷酸腺苷 $(\mathrm{ATP})^{[12]}$ )、生物大分子(如 蛋白质 ${ }^{[13]}$ 、核酸 $\left.{ }^{[14]}\right)$ 、细胞 ${ }^{[15]}$ 以及细菌 ${ }^{[16]}$, 极大地拓展 了纳米苂光探针的检测范围. 因此, 基于核酸功能化的 稀土基纳米材料构建的纳米苂光探针不仅稳定性和生 物相容性好, 并且能够极大增强与靶标物的结合能力, 从而实现在复杂生物体系中对靶标物的检测.

目前，关于稀土基纳米材料和核酸适配体在生物检 测方面的应用已有人进行了总结综述. 本课题组 ${ }^{[3 b]}$ 总 结了镧系掺杂的纳米颗粒在生物成像领域的研究进展, 谭蔚泓团队 ${ }^{[17]}$ 还介绍了核酸适配体组装的纳米材料在 生物传感和生物医学中的应用. 然而关于稀土基纳米材 料核酸功能化的综述还少有报道, 特别是其在生物检测 领域的重要潜力还需要进一步开发. 为了总结和阐明核 酸功能化稀土基纳米材料在生物检测中的重要作用, 本 综述将从核酸功能化稀土基纳米材料设计纳米荧光探 针的策略和应用方面进行综述. 首先, 介绍了几种常见 的用于设计纳米苂光探针的稀土基纳米材料. 其次, 对 核酸功能化稀土基纳米材料设计纳米苂光探针的策略 进行了归纳, 并结合一些重要工作, 进一步说明了核酸 功能化稀土基纳米材料设计纳米苂光探针的工作原理. 此外, 对基于核酸功能化稀土基纳米材料设计的纳米苂 光探针在生物检测方面的研究和应用进行了阐述. 最 后, 对核酸功能化稀土基纳米材料在生物检测领域的机
遇和挑战进行了分析和展望.

\section{2 稀土基纳米材料}

稀土基纳米材料因其独特的光学性能，作为纳米荧 光探针的发射基团在生物检测领域被广泛应用和研究. 在此, 主要介绍了稀土掺杂的上转换纳米材料 (UCNPs)、长余辉纳米材料(PLNPs)和金属有机框架化合 物(MOFs)的性质及其发光机理.

\section{1 稀土基上转换纳米材料}

稀土基 UCNPs 是一种可以将两个或两个以上的长 波长、低能量的近红外光子经非线性辐射转化为短波长 高能量光子, 从而发射出紫外/可见/近红外光的发光材 料. 这种独特的反斯托克斯发光的能力赋予了稀土基 UCNPs 背景苂光低、激发光源对生物组织的光穿透深度 高和光损伤小的优势 ${ }^{[18]}$. 稀土基 UCNPs 一般是由基质 材料、敏化剂和激活剂三部分组成. 其中激活剂作为稀 土基 UCNPs 的发光中心, 能够以苂光的形式吸收并释 放能量. 目前, $\mathrm{Er}^{3+} 、 \mathrm{Ho}^{3+}$ 和 $\mathrm{Tm}^{3+}$ 是最常见的激活剂 ${ }^{[19]}$, 分别发射绿光、红光和蓝光. 基质材料一般不构成激发 光能级, 但能够为激活离子提供合适的晶体场. 敏化剂 通常为具有较高吸收截面的稀土离子, 如 $\mathrm{Yb}^{3+}$ 和 $\mathrm{Nd}^{3+}$, 它们在吸收外界能量后可有效地将能量传递给激活剂, 增强激活剂的发光能力, 所以通过激活剂和敏化剂共掺 杂的方式能够显著提高上转换的发光效率 ${ }^{[20]}$. 掺杂在 UCNPs 中的稀土元素的丰富能级使得稀土基 UCNPs 具 有多种上转换发射的能量传递途径, 包括激发态吸收 (ESA)、能量传递上转换(ETU)以及光子雪崩(PA). 其中 激发态吸收指的是相同离子通过对多个光子的连续吸 收, 从低能量的基态向高能量的激发态跃迁的过程, 是 上转换发光的基本过程(图 1a). 能量传递上转换的过程 通常发生在两个满足能量匹配要求的不同离子之间, 其 中一个离子通过非辐射过程把能量转移给另一个离子 后回到低能态，同时另一个离子接受能量而跃迁到高能 态(图 1b). 光子雪崩机理在 1979 年对掺杂 $\operatorname{Pr}^{3+}$ 的红外计 数器研究中被首次提出 ${ }^{[21]}$, 它的机制最为复杂, 是激发 态吸收和能量传递相结合的过程. 其中能量交叉弛豫过 程的存在使得中间态粒子数雪崩式增加, 因此研究者将 这种现象称为 “光子雪崩” [20c,22]. 稀土基 UCNPs 不仅 有光穿透力强、对生物组织几乎无损伤、背景苂光低等 特点, 还具有很强的抗光漂白能力, 即使在长时间暴露 于激发辐射(长达数小时)后仍能持续发光 ${ }^{23]}$. 基于以上 优点, 稀土基 UCNPs 在 20 世纪 90 年代被应用于生物检 测的探针以来 ${ }^{[24]}$, 得到了迅速发展.

\section{2 稀土基长余辉纳米材料}

稀土基 PLNPs 是一种能够快速储存激发能(紫外 光、可见光、 $\mathrm{X}$ 射线或其他激发源的能量), 并在停止激 
(a)

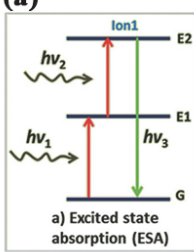

(b)

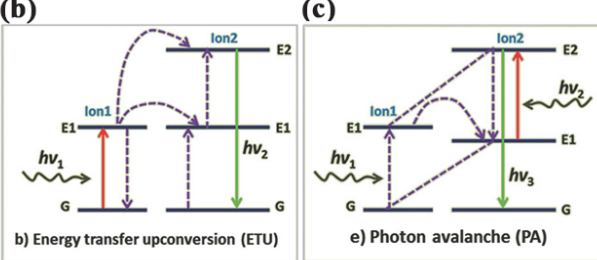

图 1 典型的上转换发光机理图: (a) 激发态吸收(ESA); (b) 能量传递 上转换; (c) 光子雪崩 ${ }^{[25]}$

Figure 1 Representative upconversion processes of (a) excited state absorption (ESA), (b) energy transfer upconversion (ETU) and (c) photon avalanche (PA). Reprinted with permission from ref. 25. Copyright 2018, Wiley-VCH Verlag GmbH \& KGaA, Weinheim.

发后长时间(几分钟、几小时甚至几天)仍能持续发光的 纳米材料. 稀土基 PLNPs 主要由陷阱中心和发射中心 组成, 部分包含传递能量的敏化剂. 稀土基 PLNPs 的陷 阱中心能够储存激发光的能量, 在受到光、热或其他方 式激活后，稀土基 PLNPs 的发射中心将储存在陷阱中 心的自由电子进行释放(图 2) ${ }^{[26]}$. 由于稀土基 PLNPs 依 靠陷阱中心储存激发能, 然后以光子发射的形式缓慢释 放能量, 所以稀土基 PLNPs 能够在成像和检测之前进 行光激发, 避免了原位激发的背景噪声, 从而提升了检 测的灵敏度和信噪比 ${ }^{[27]}$. 自从 1996 年, 一种新的稀土 基 PLNPs $\left(\mathrm{SrAl}_{2} \mathrm{O}_{4}: \mathrm{Eu}^{2+}{ }^{2} \mathrm{Dy}^{3+}\right)$ 被报道 ${ }^{[28]}$ 后，稀土基 PLNPs 长寿命的发光得到了广泛关注和迅速的发展, 许 多不同基质和掺杂不同稀土离子的 PLNPs 被报道出来. Scherman 和他的同事 ${ }^{[29]}$ 在 2007 年第一次将发射近红外 光的稀土基 PLNPs $\left(\mathrm{MgSiO}_{3}: \mathrm{Eu}^{2+}, \mathrm{Dy}^{3+}, \mathrm{Mn}^{2+}\right)$ 用于活体 动物的实时光学成像, 克服了传统荧光探针信噪比低、 深部组织成像困难的缺点, 为小鼠肿瘤成像和治疗评价 提供了重要的帮助. 稀土元素被掺杂在 PLNPs 中可作 为敏化剂或发射中心, 以实现稀土基 PLNPs 从紫外到 近红外区域的持久发光 ${ }^{\left[{ }^{[b]}\right.}$. 江国健课题组 ${ }^{[30]}$ 通过对稀 土离子 $\left(\mathrm{Pr}^{3+} 、 \mathrm{Nd}^{3+} 、 \mathrm{Gd}^{3+}\right)$ 掺杂的 $\mathrm{Li}_{2} \mathrm{ZnGeO}$ 余辉性能的 研究, 证明了稀土掺杂的 PLNPs 缺陷密度值较大, 余辉 持久性更强, $\mathrm{Gd}^{3+} 、 \mathrm{Pr}^{3+}$ 和 $\mathrm{Nd}^{3+}$ 的掺杂分别使 $\mathrm{Li}_{2} \mathrm{ZnGeO}_{4}$ 的荧光寿命从 $83.35 \mathrm{~ns}$ 提升到了 89.66 、94.93 和 $95.41 \mathrm{~ns}$. 此外, 很多研究者通过稀土离子的掺杂还研制出了具有 上转换功能的长余辉材料, 这些材料结合了上转换反斯 托克斯发光和长余辉持续发光的优点, 能够产生发射能 量高于激发能量的持续发光, 是制备光学探针理想的材 料 ${ }^{[31]}$. 如孙康宁课题组 ${ }^{[31 a]}$ 通过对 $\mathrm{Zn}_{3} \mathrm{Ga}_{2} \mathrm{SnO}_{8}: \mathrm{Cr}^{3+}$ 进行 $\mathrm{Yb}^{3+}, \mathrm{Er}^{3+}$ 的掺杂, 使得该荧光粉在近红外光激发下表 现出了上转换的持久发光. 稀土基 PLNPs 无背景苂光 且余辉寿命长的特点使其在生物医学领域, 特别是生物 检测领域的应用越来越多.

\section{3 稀土基金属有机框架化合物}

稀土基 MOFs 是一种新型多孔材料, 由稀土离子/ 簇和多齿有机连接基通过配位作用组装而成. MOFs 通

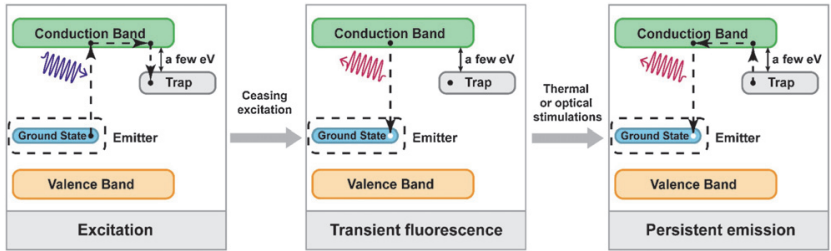

图 2 稀土基 PLNPs 的持久发光原理示意图 ${ }^{[26]}$.

Figure 2 Schematic illustration of persistent luminescence mechanisms of rare earth PLNPs. Reprinted with permission from ref. 26. Copyright 2019, Chinese Chemical Society and Institute of Materia Medica, Chinese Academy of Medical Sciences.

常具有可调节的结构与功能, 较大的比表面积和高度有 序的多孔结构, 这些特点使其广泛应用于气体吸附与分 离、催化、传感、药物递送和质子传导等领域 ${ }^{[32]}$. 由于 稀土离子通常通过能量/电子转移过程被周围的配体激 发, 稀土基 MOFs 中的有机配体在吸收激发光的能量后, 通过非辐射过程把能量转移到稀土离子, 从而可以产生 发光现象. 因此，以稀土离子为金属节点的 MOFs 在光 学传感领域的应用也逐渐成为了研究的热点 ${ }^{[14,33]}$. 由于 结构和功能的可调性，通过对稀土基 MOFs 中的稀土离 子和有机配体的调节设计的稀土基 MOFs 具有良好的发 光特性 ${ }^{[34]} .2007$ 年, Rieter 和同事 ${ }^{[35]}$ 首次证明了稀土基 MOFs 可以用于二吡啶酸的检测，表明了稀土基 MOFs 在构建纳米苂光探针并应用于生物检测领域的潜力. 此 外，作为纳米荧光探针发光基团的稀土基 MOFs 还具备 增强纳米苂光探针发光强度、降低检测限的特点. 该特 点主要来源于以下两个原因: 其一, 由于稀土离子嵌入 MOFs 高度有序的有机配体框架中避免了由于聚集引起 的自猝灭，从而使稀土基 MOFs 的发光增强 ${ }^{[36]}$. 其二, 稀土基 MOFs 的高比表面积能够实现核酸适配体的高负 载, 从而显著增加与靶标物结合的有效活性位点的数 量，促进核酸适配体与靶标物结合的效率，达到降低纳 米荧光探针的检测限的效果，使纳米荧光探针的传感性 能得到提升 ${ }^{[14]}$. 总之, 基于稀土基 MOFs 设计的纳米荧 光探针在生物检测领域具有巨大潜力, 并在提升纳米苂 光探针性能方面有着独特的优势.

\section{3 核酸功能化稀土基纳米材料设计纳米荧光探 针的策略}

核酸适配体作为一种能与靶标物特异性结合的识 别元件，能够赋予稀土基纳米材料强大的特异性识别能 力以及生物相容性，有效提升探针的检测效率. 此外, 核酸适配体独特的空间构象变化以及碱基编码方式，为 纳米荧光探针的响应策略设计提供了许多思路，从而让 纳米苂光探针非常 “聪明” 地发现靶标物并产生光学响 应，极大地丰富了稀土基纳米荧光探针的种类和检测范 围. 目前, 大部分的核酸功能化纳米苂光探针主要使用 苂光强度作为定量分析的信号模式实现高灵敏度荧光 测量 ${ }^{[37]}$. 下面我们重点讨论了以核酸适配体为识别元 
件、稀土基纳米材料作为苂光团, 及以苂光强度作为检 测信号的纳米苂光探针的设计策略, 并将设计策略分为 了以下三种模式: 构象转换模式、“三明治” 模式和竞 争替代模式.

\section{1 构象转换模式}

为了与靶标物更好地结合, 核酸适配体通常会通过 互补碱基配对、静电作用或氢键等作用力, 折叠形成发 卡、假结、茎环、G-四链体等稳定的三维空间结构 ${ }^{[38]}$. 构 象转换模式即基于核酸适配体构象发生特定变化而引 起检测信号改变的模式.

在这种模式的策略下，核酸适配体两端通常连接苂 光供体和受体, 并且两者的相对位置可以决定荧光共振 能量转移(FRET)效率, 从而改变稀土基纳米材料的发 光效果. 基于此, 鞠熀先课题组 ${ }^{[39]}$ 通过对核酸适配体两 端连接的苂光供体稀土基 PLNPs 和受体分子异硫氰酸 荧光素(FITC)距离的控制, 成功实现了稀土基 PLNPs 和 FITC 之间时间分辨苂光共振能量转移(TR-FRET)效率 的转变. 该设计原理基于 FITC 的荧光寿命比延迟时间 (脉冲激发光到信号采集之间的一段时间)短从而苂光得 到了有效抑制的原理, 利用核酸适配体对靶标物特异性 结合后, 其结构发生了转换从而拉近了稀土基 PLNPs 与 FITC 的距离, 使得稀土基 PLNPs 最大发射波长和 FITC 最大激发波长的重叠并导致 TR-FRET 效率增强 (图 3). 他们进一步将 FITC 标记的核酸适配体对稀土基 PLNPs 进行功能化构建了纳米菼光探针, 成功实现了血 小板源生长因子的生物检测.
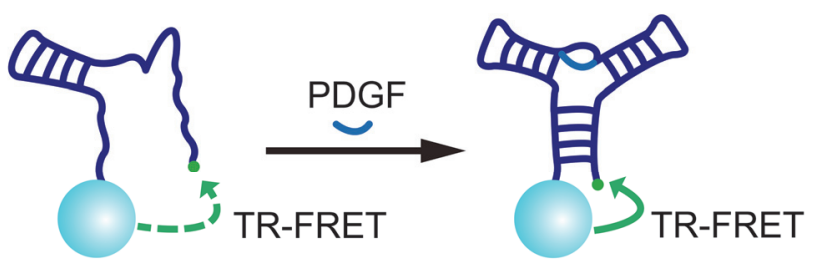

\section{Aptamer-PLNP}

图 3 核酸适配体与靶标物结合引起信号基团相对距离变化的示意 图.

Figure 3 Scheme of the relative distance changes of signal groups after aptamers binding targets.

此外, 靶标物与核酸适配体特异性结合产生的稳定 三维构象也会导致核酸适配体性能的变化. 张志琪研究 团队 ${ }^{[40]}$ 首次证明了 ATP 与核酸适配体形成的 G-四链体 比核酸适配体单链对金纳米粒子(AuNPs)的稳定性更强, 能够防止 AuNPs 在高盐浓度下的聚集. 在此基础上, 尤 进茂团队 ${ }^{[12]}$ 以 AuNPs 作为 Tb 掺杂的 MOFs (Tb-MOFs) 的猝灭剂, 进一步利用了团聚和分散情况下的 AuNPs 对 Tb-MOFs 的荧光猝灭效果的差距设计了用于检测 ATP 的纳米苂光探针. 在未出现靶标物 ATP 的情况下, 自动聚集的 AuNPs 几乎不能猝灭 Tb-MOFs 的苂光. 而
ATP 出现后, 其与核酸适配体结合形成的 G-四链体为 AuNPs 提供了良好的胶体稳定效果，分散的 AuNPs 对 Tb-MOFs 苂光的猝灭使得荧光信号减弱, 从而实现了 ATP 的高效检测.

\section{2 “三明治” 模式}

“三明治” 模式通常以靶标物为中心，靶标物同时 结合两个核酸适配体, 并进一步拉近两个核酸适配体各 自连接的荧光供体与受体的距离, 从而形成 “夹心” 结 构. 所以，这种模式要求靶标物具有双结合位点，形成 “三明治” 结构的核酸适配体则可以是相同的也可以是 不同的. 研究者们成功运用 “三明治” 模式构建了检测 蛋白、核酸以及细菌的纳米苂光探针.

一些蛋白质，如血小板源性生长因子和凝血酶具有 双重结合位点，能够结合两个识别分子从而形成 “三明 治” 复合体. “核酸适配体-凝血酶-核酸适配体” 的 “三 明治模式”已经被报道用于凝血酶的高特异性检测. 王 伦课题组 ${ }^{[41]}$ 设计了一种选择性测定凝血酶的 FRET 系统, 其中核酸功能化的稀土基 UCNPs (TBA15-UCNPs)作为 供体, 核酸功能化的金纳米棒(AuNPs-TBA29)作为受体. 由于 TBA15 和 TBA29 两种核酸适配体不互补, 在没有 凝血酶的情况下, TBA15-UCNPs 无法捕获 AuNPsTBA29, 呈两种独立且分散的探针. 一旦凝血酶出现, 两个 TBA 即分别形成分子间 G-四分体结构后识别凝血 酶的不同活性位点. 这种特异性结合将供体和受体的距 离拉近，从而导致了 FRET 的发生以及稀土基 UCNPs 苂光猝灭. 寡核苷酸与相应核酸适配体的结合也被用于 “三明治” 模式的设计，它的实质是通过寡核苷酸与核 酸适配体碱基互补配对形成氢键. 由于核酸适配体合成 的可控性，靶标寡核苷酸可以结合多段核酸适配体序列， 因此 “三明治” 模式可以很容易实现寡核苷酸的检测. Afzalinia 等 ${ }^{[14]}$ 以 microRNA (miRNA)-155 互补的两段核 酸适配体序列作为识别单元, 采用 La-MOF 和金纳米粒 子(AgNPs) 作为苂光猝灭过程中的能量供 体-受体对, 实现了对 miRNA-155 的高灵敏度检测(图 4).

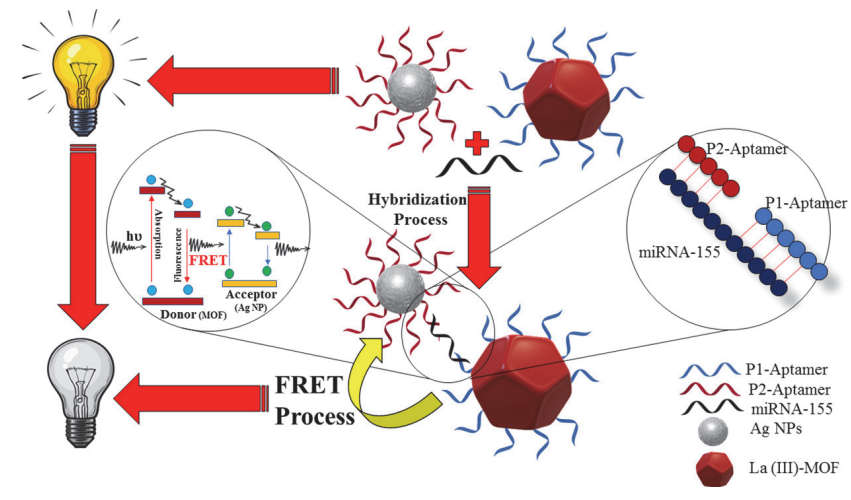

图 4 用于 RNA 检测的 “三明治” 模式设计示意图 ${ }^{[14]}$.

Figure 4 Schematic illustration of sandwich mode for RNA detection. Reprinted with permission from ref. 14. Copyright 2020, American Chemical Society. 
核酸适配体对细胞和细菌等复杂结构的识别依赖 于与其生物标志物, 如蛋白质、多聚糖等的结合. 这些 生物标志物作为检测细胞和细菌的靶标物, 使得细胞和 细菌具有多个核酸适配体的结合位点, 从而实现 “三明 治” 模式的检测. 王周平课题组 ${ }^{[42]}$ 基于 “核酸适配体细菌-磁性纳米颗粒” 的 “三明治” 模式，构建了同时检 测伤寒沙门氏菌和金黄色葡萄球菌的光学生物传感器. 研究中用两种细菌的特异性核酸适配体分别修饰了 $\mathrm{NaYF}_{4}, \mathrm{Yb} / \mathrm{Tm}$ UCNPs 和 $\mathrm{NaYF}_{4}, \mathrm{Yb} / \mathrm{Er}$ UCNPs, 并制备 了核酸适配体修饰的磁性纳米颗粒(MNPs). 在存在伤 寒沙门氏菌或金黄色葡萄球菌的情况下, 核酸适配体修 饰的稀土基 UCNPs 与核酸适配体修饰的 MNPs 形成具 有磁性的纳米颗粒生物复合物, 最后, 利用磁铁将两种 细菌的三明治复合物浓缩并分离. 在 $980 \mathrm{~nm}$ 激光的激 发下, 通过检测两种 UCNPs 在不同发射波段的苂光强 度从而在水溶液实现了对两种不同类型细菌的定性及 定量检测, 同时该设计也为生物体系内细菌和细胞的检 测提供了参考.

\section{3 竞争替代模式}

核酸适配体作为识别元件对靶标物具有高亲和性. 因此, 基于靶标物与核酸适配体更强的亲和力, 取代与 核酸适配体结合较弱的结合物, 从而设计具有高特异性 的苂光传感器也是一种常用的策略, 我们把这种策略称 为竞争替代模式. 核酸适配体与靶标物的亲和性高于与 其结合物的亲和性是该竞争性取代的关键. 目前已经报 道的作为核酸适配体结合物的有 DNA 链、阳离子聚合 物、碳纳米粒子等. 如图 $5 \mathrm{a}$ 所示, 在 $\mathrm{Au}-\mathrm{Au}$-稀土基 UCNPs 三聚体的设计实现了对甲胎蛋白(AFP)和黏蛋白 1 (mucin-1) 的双模式检测 ${ }^{[43]}$. 在该三聚体中, 两个 AuNPs 的 “热点” 现象导致了拉曼信标产生了较高的拉 曼信号强度, 同时稀土基 UCNPs 的发光强度被附近的 AuNPs 抑制. 然而, 在 AFP 和 mucin-1 靶点存在的情况 下，它们与各自对应的核酸适配体的互补 DNA 竞争, 导致 $\mathrm{Au}-\mathrm{Au}$-稀土基 UCNPs 三聚体的解离, 引起了稀土 基 UCNPs 发光强度增强和信标拉曼信号强度减弱, 从 而实现两种靶标物的灵敏检测. 王硕课题组 ${ }^{[44]}$ 同样基 于 DNA 链的竞争替代, 将核酸适配体修饰的 PLNPs 和 互补 DNA 链修饰的 $\mathrm{CuS}$ 结合, 在检测过程中靶标物与 核酸适配体的高亲和性导致其与互补 DNA 的解离, 从 而使得猝灭剂 $\mathrm{CuS}$ 与 PLNPs 的分离, 失去猝灭效果. 在 庞代文课题组 ${ }^{[45]}$ 设计的荧光传感器(图 5b)中, 首先利用 凝血酶的核酸适配体共价结合丙烯酸功能化的稀土基 上转换荧光粉(UCPs), 核酸适配体通过 $\pi-\pi$ 堆积作用与 碳纳米颗粒(CNPs) 相连, 由于 FRET 作用, UCPs 的发光 被猝灭. 凝血酶靶标物的出现诱导核酸适配体形成 G四链体结构, 使得核酸适配体与 CNPs 之间的 $\pi-\pi$ 作用 减弱, 最终导致 CNPs 被释放, UCPs 恢复发光, 从而达
到检测凝血酶的目的. 朱俊杰课题组 ${ }^{[46]}$ 通过阳离子聚 合物作为静电连接剂将合成的稀土基 UCNPs 与染料分 子标记的核酸适配体连接, 稀土基 UCNPs 和染料分子 之间的 FRET 作用可以导致上转换发光强度降低. 在溶 菌酶或互补 DNA 的存在下, 由于其与核酸适配体具有 较强的结合作用, 核酸适配体从阳离子聚合物上解离下 来，使稀土基 UCNPs 脱离了与苂光染料发生 FRET 的有 效距离, 导致稀土基 UCNPs 的发光强度得到恢复.
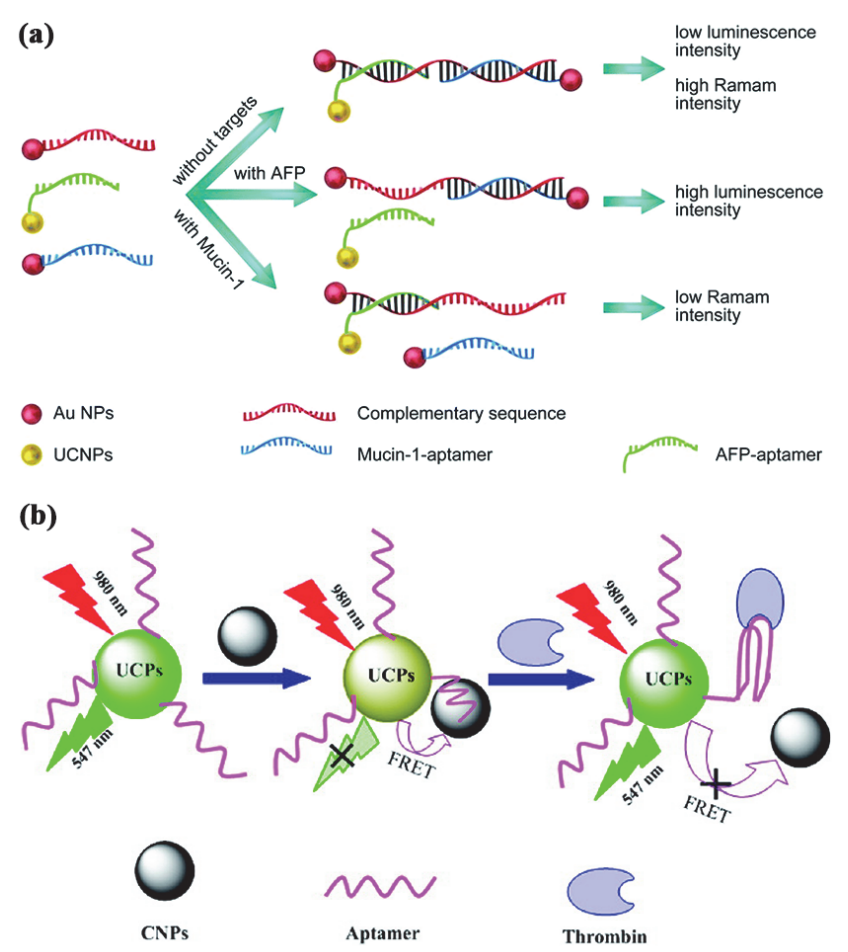

图 5 竞争替代模式的设计示意图. 靶标物与(a)互补 DNA 链 ${ }^{[43]}$ 和(b) 碳纳米颗粒 $(\mathrm{CNPs})^{[45]}$ 竞争结合核酸适配体.

Figure 5 Schematic diagram of competitive replacement mode. Competition of targets with (a) DNA strands ${ }^{[3]}$ and (b) carbon nanoparticles $(\mathrm{CNPs})^{[45]}$. Reprinted with permission from ref. 43 (Copyright 2017, The Royal Society of Chemistry) and ref. 45 (Copyright 2011, American Chemical Society).

\section{4 其他}

除这些常用模式之外, 核酸适配体还可以用于分子 印迹技术. 将核酸适配体设计为分子印迹聚合物作为识 别元件, 可以打破单一识别的方式, 实现对靶标物的双 重识别, 从而达到提高识别效率和准确率的目的. 励建 荣课题组 ${ }^{[47]}$ 将恩罗沙星(ENR)核酸适配体固定在稀土基 UCNPs 的表面用于捕获 ENR, 形成第一个印迹识别保 障. 再利用丙烯酸单体与 ENR 的残基相互作用, 形成第 二个印迹识别保障. 去除 ENR 后形成的 “双重识别” 印 迹空腔表现出了优于核酸适配体和传统分子印迹的识 别特性，并且在该纳米荧光探针识别并结合 ENR 后，稀 土基 UCNPs 的苂光被猝灭(图 6). 


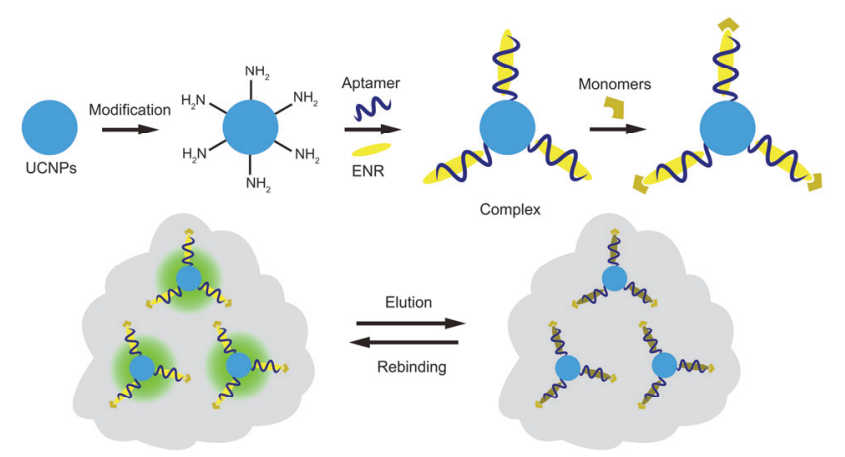

图 6 基于核酸适配体的分子印迹聚合物的制备和识别过程示意图. Figure 6 Preparation of molecularly imprinted polymers based on aptamers and subsequent identification process.

\section{4 基于核酸功能化稀土基纳米材料的纳米荧光 探针在生物检测中的应用}

疾病生物标志物的检测对于疾病的诊断、治疗和预 后都至关重要, 如核酸、蛋白质、细胞等生物标志物能 够揭示多种疾病进程, 为及时有效的治疗提供重要参 考. 此外, 细菌和一些生物小分子及离子含量对人体健 康产生的影响也不可忽视, 如录离子对泌尿系统、生殖 系统和消化系统都会产生毒性 ${ }^{[48]}$. 因此, 体内这些生物 分子的检测对人体健康的意义重大. 在本节中, 我们将 重点讨论基于核酸功能化稀土基纳米材料设计的纳米 苂光探针在各类疾病生物标志物的检测中的应用(图 7).

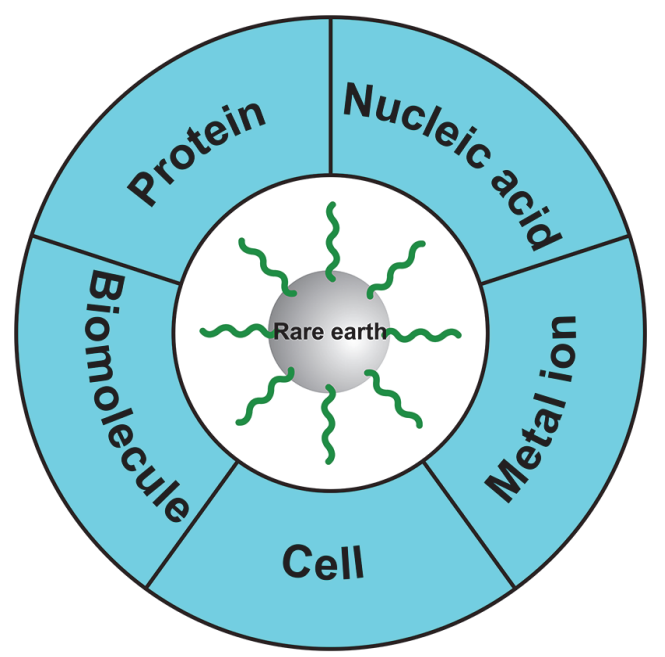

图 7 核酸功能化稀土基纳米材料用于生物检测的示意图.

Figure 7 Scheme of aptamer-functionalized rare earth nanomaterials for bioassays.

\section{1 核酸检测}

核酸分子是生物体的基本构成物质和基因表达的 基础, 可以在分子生物学、疾病诊断和治疗等领域提供 足够的信息. 基因突变是引起部分疾病的原因, 疾病的 发展也可能会导致某些核酸的异常编码, 因此在分子水 平上对基因突变的检测可以实现疾病在无症状阶段的
诊断. 此外，对核酸的检测还能够深化我们对基因表达 调控的认识 ${ }^{[49]}$. 最近的研究表明, 基于 La-MOF 和 AuNPs 的超敏荧光 miRNA 生物传感器在早期癌症诊断 发挥了重要作用, 证明了 miRNA-155 在乳腺癌和肺癌 患者的生物体液中异常表达, 该工作在肺癌和乳腺癌的 诊断中表现出了巨大潜力 ${ }^{[14]}$.

\section{2 蛋白质检测}

蛋白质是生命活动的基础, 它不仅是构成细胞和生 物体结构的重要物质, 还具有催化生理反应、运输营养 物质、参与免疫反应和传递信息等功能. 因此对蛋白质 的检测有助于我们更好地理解生命活动的内在机制, 对 疾病特异性蛋白生物标志物的检测更有助于疾病的早 期诊断、预防及监控. 以核酸功能化稀土基纳米材料为 苂光团构建的纳米苂光探针已经实现了多种蛋白质, 包

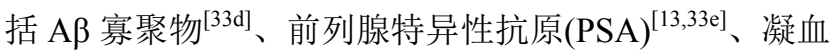
酶 ${ }^{[13,41]}$ 、溶菌酶、血小板源生长因子 ${ }^{[39]}$ 、甲胎蛋白 $(\mathrm{AFP})$ 和黏蛋白 1 (mucin-1) $)^{[43]}$ 等蛋白质的检测. 其中, $\mathrm{A} \beta$ 寡聚 物、PSA、AFP 的成功检测分别能够为阿尔兹海默症、 前列腺癌和原发性肝癌的诊断提供依据. mucin-1 和溶 菌酶的表达异常还与多种疾病或癌症有关. 此外, 本课 题组 ${ }^{[50]}$ 还通过构建用于指纹中溶菌酶检测的探针实现 了指纹成像, 拓展了蛋白质检测刑事侦查领域的应用.

\section{3 细胞和细菌检测}

与核酸和蛋白质等生物标志物相比, 肿瘤细胞具有 更大的表面积和更多的结合位点. 多种针对不同细胞类 型的核酸适配体已经被开发, 为实际血液样本中癌细胞 的直接检测提供了可能性 ${ }^{[49 a]}$. 循环肿瘤细胞(CTC)的检 测在肿瘤的诊断和预后中起着重要的作用, 核酸功能化 的稀土基 UCNPs 作为纳米探针已经被设计用于 CTC 检 测, 并实现了血液样本中的检测, 展现了稀土基纳米苂 光探针在 CTC 检测中的临床应用前景. 与细胞的检测 类似，细菌同样具有多重结合位点，细菌的快速、灵敏 检测在生物技术、医学诊断、食品安全等领域都具有极 其重要的意义. 基于核酸功能化稀土基纳米材料设计的 纳米荧光探针已经实现了溶液体系中大肠杆菌 ${ }^{[16]}$ 、沙门 氏菌和金黄色葡萄球菌的同时检测 ${ }^{[42]}$, 为生物体系中 细菌的检测开辟了道路.

\section{4 生物小分子及金属离子检测}

生物体内一些小分子及金属离子的含量对生物体 的影响也不可忽视, 例如, ATP 作为生命的主要能量来 源, 参与各种细胞代谢过程的调节, 在疾病监测中也发

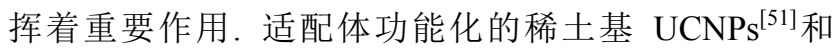
Tb-MOFs 纳米苂光探针 ${ }^{[12]}$ 已经实现了 ATP 的检测. 通 过食物链进入人体的抗菌剂恩诺沙星 $(\mathrm{ENR})^{[47]}$ 、真菌毒 素 ${ }^{[52]} 、 \mathrm{Hg}^{2+[53]}$ 和 $\mathrm{Pb}^{2+[53 b, 54]}$ 等可能会对人体健康产生多 种副作用, 基于核酸功能化稀土基纳米材料设计的纳米 荧光探针为这些小分子或离子在溶液体系的检测提供 
了方法, 有望进一步实现在生物体系的检测. 总之, 对 这些生物小分子及金属离子的检测对疾病预防与诊断、 食品分析等领域都具有重要作用.

\section{5 总结}

由于稀土基纳米材料发射线尖锐、斯托克斯位移/ 反斯托克斯位移大、苂光寿命长、量子产率高等特性和 核酸适配体识别能力强、生物安全性高等优势, 基于核 酸功能化稀土基纳米材料构建的纳米荧光探针在生物 检测领域得到了广泛研究. 本综述主要对稀土基纳米材 料的常见种类、核酸功能化稀土基纳米材料设计纳米苂 光探针的策略及检测物质分类进行了总结. 核酸功能化 稀土基纳米材料在蛋白质、核酸、细胞结构、生物小分 子及离子的应用场景中都实现了高灵敏度、准确有效的 检测. 核酸功能化的稀土基纳米材料稳定性和灵敏度高 并且识别能力强, 过去十年间在生物检测领域的应用非 常广泛. 尽管这一领域目前已经取得了巨大的进展, 但 未来还有许多工作需要进一步开展:

(1)丰富稀土基纳米材料的种类. 目前在生物检测 领域中, 被核酸功能化的稀土基纳米材料以稀土基 UCNPs 为主. 稀土基 PLNPs 避免原位激发、发光寿命 长的优势和稀土基 MOFs 有序多孔结构防止自猝灭以及 核酸适配体负载量高的特点, 对实现稳定高效的生物检 测具有重要意义, 然而对稀土基 PLNPs 和稀土基 MOFs 的研究还不够普遍, 未来应当设计开发更多稀土基纳米 材料, 包括稀土基 PLNPs、稀土基 MOFs, 另外还有稀 土掺杂的量子点 ${ }^{[5]}$ 等新型材料, 丰富核酸功能化的稀 土基纳米材料的种类及其功能.

(2)拓展核酸功能化稀土基纳米材料的应用场景. 基于核酸功能化稀土基纳米材料设计的纳米苂光探针 对细菌以及金属离子的检测还局限于水溶液体系, 如对 恩诺沙星、真菌毒素、 $\mathrm{Hg}^{2+}$ 和 $\mathrm{Pb}^{2+}$ 的检测还未深入到生 物体系. 在复杂生物样本和活体内这些物质的检测还较 为缺乏, 这类研究更为复杂同时也对生物检测的实际应 用至关重要. 未来需要进一步探索在生物体系的原位检 测方法, 将检测技术推行到临床应用当中.

(3)将其他传感技术与核酸适配体结合, 开发识别 性能更好的苂光传感器. 基于分子印迹技术的核酸功能 化上转换纳米材料设计的苂光传感器在对靶标物的识 别效率和准确率上都实现了突破. 将核酸功能化稀土基 纳米材料与其他传感技术结合开发新型纳米苂光传感 器将是一个重要的研究方向, 有望解决复杂体系检测中 效率较低等问题.

总之, 基于核酸功能化稀土基纳米材料设计的纳米 荧光探针在生物检测领域具有广阔的前景, 并可能在人 体健康情况的监测方面发挥更大的作用, 但目前这一领 域的材料还相对局限, 有进一步开发探索的空间, 检测 范围也有待进一步扩展, 不仅需要拓宽检测物质的种
类, 更需要深入探究对生物体系的原位实时检测. 随着 相关研究的不断深入, 相信核酸功能化稀土基纳米材料 在生物检测领域将实现更加广泛和深入的应用.

\section{作者简介}

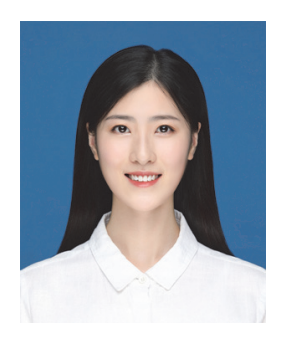

贾伊祎, 2018 年于华中农业大学理学院获得学士学位, 同 年加入湖南大学化学化工学院攻读硕士学位, 主要研究兴趣 为金属框架化合物在生物医学中的应用.

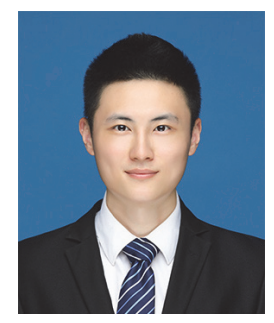

王文杰, 2018 年于华北电力大学(保定)环境科学与工程学 院获得学士学位, 同年加入湖南大学化学化工学院攻读硕士 学位, 主要研究兴趣为无机纳米材料在生物医学中的应用.

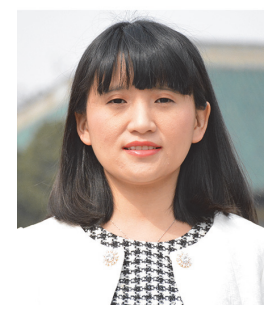

袁荃, 教授, 2004 年于武汉大学获学士学位, 2009 年于北 京大学获博士学位, 2009 年至 2012 年在佛罗里达大学谭蔚泓 课题组进行博士后研究. 袁荃教授目前在湖南大学化学化工 学院进行研究工作, 主要研究兴趣包括功能纳米材料的可控 合成及其在生物医学中的应用.

\section{References}

[1] Pehlivan, Z. S.; Torabfam, M.; Kurt, H.; Ow-Yang, C.; Hildebrandt, N.; Yüce, M. Microchim. Acta 2019, 186, 563.

[2] Escudero, A.; Becerro, A. I.; Carrillo-Carrión, C.; Núñez, N. O.; Zyuzin, M. V.; Laguna, M.; González-Mancebo, D.; Ocaña, M.; Parak, W. J. Nanophotonics 2017, 6, 881.

[3] (a) Wang, G. F.; Peng, Q.; Li, Y. D. Acc. Chem. Res. 2011, 44, 322; (b) Ma, Q. Q.; Wang, J.; Li, Z. H.; Lv, X. B.; Liang, L.; Yuan, Q. Small 2019, 15, 1804969; (c) Xiong, L.; Fan, Y.; Zhang, F. Acta Chim. Sinica 2019, 77, 1239 (in Chinese). (熊麟, 凡勇, 张凡, 化 学学报, 2019, 77, 1239.)

[4] Kuningas, K.; Ukonaho, T.; Päkkilä, H.; Rantanen, T.; Rosenberg, J.; Lövgren, T.; Soukka, T. Anal. Chem. 2006, 78, 4690.

[5] Ma, L.; Liu, F. Y.; Lei, Z.; Wang, Z. X. Biosens. Bioelectron. 2017, $87,638$.

[6] Saleh, S. M.; Ali, R.; Hirsch, T.; Wolfbeis, O. S. J. Nanopart. Res. 2011, 13, 4603 . 
[7] Wang, Y. H.; Shen, P.; Li, C. Y.; Wang, Y.; Liu, Z. Y. Anal. Chem. 2012, 84, 1466.

[8] Tuerk, C.; Gold, L. Science 1990, 249, 505.

[9] Ellington, A. D.; Szostak, J. W. Nature 1990, 346, 818.

[10] Yüce, M.; Ullah, N.; Budak, H. Analyst 2015, 140, 5379.

[11] Tu, J. W.; Gan, Y.; Liang, T.; Wang, Q.; Ren, T. L.; Sun, Q. Y.; Wan, H.; Wang, P. Front. Chem. 2018, 6, 333.

[12] Qu, F.; Sun, C.; Lv, X. X.; You, J. M. Microchim. Acta 2018, 185, 359.

[13] Hao, T. T.; Wu, X. L.; Xu, L. G.; Liu, L. Q.; Ma, W.; Kuang, H.; Xu, C. Small 2017, 13, 1603944.

[14] Afzalinia, A.; Mirzaee, M. ACS Appl. Mater. Interfaces 2020, 12 , 16076.

[15] Bashmakova, E. E.; Krasitskaya, V. V.; Zamay, G. S.; Zamay, T. N.; Frank, L. A. Talanta 2019, 199, 674.

[16] Jin, B.; Wang, S.; Lin, M.; Jin, Y.; Zhang, S.; Cui, X.; Gong, Y.; Li, A.; Xu, F.; Lu, T. J. Biosens. Bioelectron. 2017, 90, 525.

[17] Kong, R. M.; Zhang, X. B.; Chen, Z.; Tan, W. Small 2011, 7, 2428.

[18] Wang, F.; Banerjee, D.; Liu, Y. S.; Chen, X. Y.; Liu, X. G. Analyst 2010, 135, 1839

[19] (a) Ang, L. Y.; Lim, M. E.; Ong, L. C.; Zhang, Y. Nanomedicine 2011, 6, 1273; (b) Chen, J.; Zhao, J. X. Sensors 2012, 12, 2414.

[20] (a) Heer, S.; Lehmann, O.; Haase, M.; Güdel, H. U. Angew. Chem., Int. Ed. 2003, 42, 3179; (b) Lin, M.; Zhao, Y.; Wang, S. Q.; Liu, M.; Duan, Z. F.; Chen, Y. M.; Li, F.; Xu, F.; Lu, T. J. Biotechnol. Adv. 2012, 30, 1551; (c) DaCosta, M. V.; Doughan, S.; Han, Y.; Krull, U. J. Anal. Chim. Acta 2014, 832, 1.

[21] Chivian, J. S.; Case, W. E.; Eden, D. D. Appl. Phys. Lett. 1979, 35, 124.

[22] Hong, E.; Liu, L. M.; Bai, L. M.; Xia, C. H.; Gao, L.; Zhang, L. W.; Wang, B. Q. Mater. Sci. Eng., C 2019, 105, 110097.

[23] Auzel, F. Chem. Rev. 2004, 104, 139.

[24] Yao, C. Z.; Yao, C. Z.; Tong, Y. X. TrAC, Trends Anal. Chem. 2012, $39,60$.

[25] Lingeshwar Reddy, K.; Balaji, R.; Kumar, A.; Krishnan, V. Small 2018, 14, 1801304

[26] Lin, Q. S.; Li, Z. H.; Yuan, Q. Chin. Chem. Lett. 2019, 30, 1547.

[27] (a) Liu, Y. L.; Kuang, J. Y.; Lei, B. F.; Shi, C. S. J. Mater. Chem. 2005, 15, 4025; (b) Pan, Z. W.; Lu, Y. Y.; Liu, F. Nat. Mater. 2011, 11, 58; (c) Maldiney, T.; Bessière, A.; Seguin, J.; Teston, E.; Sharma, S. K.; Viana, B.; Bos, A. J. J.; Dorenbos, P.; Bessodes, M.; Gourier, D.; Scherman, D.; Richard, C. Nat. Mater. 2014, 13, 418.

[28] Matsuzawa, T.; Aoki, Y.; Takeuchi, N. J. Electrochem. Soc. 1996, $143,2670$.

[29] le Masne de Chermont, Q.; Chaneac, C.; Seguin, J.; Pelle, F.; Maitrejean, S.; Jolivet, J. P.; Gourier, D.; Bessodes, M.; Scherman, D. Proc. Nat. Acad. Sci. 2007, 104, 9266.

[30] Tu, T. Z.; Jiang, G. J. J. Mater. Sci.: Mater. Electron. 2018, 29, 3146.

[31] (a) Ge, P. H.; Sun, K. N.; Cheng, Y. Optik 2019, 188, 200; (b) Liu, F.; Liang, Y. J.; Pan, Z. W. Phys. Rev. Lett. 2014, 113, 177401; (c) Li, Z. J.; Huang, L.; Zhang, Y. W.; Zhao, Y.; Yang, H.; Han, G. Nano Res. 2017, 10, 1840; (d) Xue, Z. L.; Li, X. L.; Li, Y. B.; Jiang, M. Y.; Ren, G. Z.; Liu, H. R.; Zeng, S. J.; Hao, J. H. Nanoscale 2017, 9, 7276.

[32] (a) Zhou, H. C. J.; Kitagawa, S. Chem. Soc. Rev. 2014, 43, 5415; (b) Ding, M.; Cai, X.; Jiang, H. L. Chem. Sci. 2019, 10, 10209.

[33] (a) Zhang, S. Y.; Shi, W.; Cheng, P.; Zaworotko, M. J. J. Am. Chem.
Soc. 2015, 137, 12203; (b) Wu, S. Y.; Lin, Y. N.; Liu, J.; Shi, W.; Yang, G. M.; Cheng, P. Adv. Funct. Mater. 2018, 28, 1707169; (c) Xia, C.; Xu, Y.; Cao, M. M.; Liu, Y. P.; Xia, J. F.; Jiang, D. Y.; Zhou, G. H.; Xie, R. J.; Zhang, D. F.; Li, H. L. Talanta 2020, 212, 120795 ; (d) Ren, H. X.; Miao, Y. B.; Zhang, Y. Microchim. Acta 2020, 187, 114; (e) Qu, F.; Ding, Y. R.; Lv, X. X.; Xia, L.; You, J. M.; Han, W. L. Anal. Bioanal. Chem. 2019, 411, 3979.

[34] Cui, Y. J.; Zhang, J.; He, H. J.; Qian, G. D. Chem. Soc. Rev. 2018, 47,5740 .

[35] Rieter, W. J.; Taylor, K. M. L.; Lin, W. J. Am. Chem. Soc. 2007, 129, 9852.

[36] Mahata, P.; Mondal, S. K.; Singha, D. K.; Majee, P. Dalton Trans. 2017, 46, 301.

[37] Juskowiak, B. Anal. Bioanal. Chem. 2011, 399, 3157.

[38] (a) Sakamoto, T.; Ennifar, E.; Nakamura, Y. Biochimie 2018, 145, 91; (b) Zhou, J. H.; Rossi, J. Nat. Rev. Drug Discovery 2017, 16, 181.

[39] Zhang, L.; Lei, J. P.; Liu, J. T.; Ma, F. J.; Ju, H. X. Biomaterials 2015, 67, 323.

[40] Huo, Y.; Qi, L.; Lv, X. J.; Lai, T.; Zhang, J.; Zhang, Z. Q. Biosens. Bioelectron. 2016, 78, 315.

[41] Chen, H. Q.; Yuan, F.; Wang, S. Z.; Xu, J.; Zhang, Y. Y.; Wang, L. Biosens. Bioelectron. 2013, 48, 19.

[42] Duan, N.; Wu, S. J.; Zhu, C. Q.; Ma, X. Y.; Wang, Z. P.; Yu, Y.; Jiang, Y. Anal. Chim. Acta 2012, 723, 1.

[43] Qu, A. H.; Wu, X. L.; Xu, L. G.; Liu, L. Q.; Ma, W.; Kuang, H.; Xu, C. L. Nanoscale 2017, 9, 3865.

[44] Liu, J. M.; Yuan, X, Y.; Liu, H, L.; Cheng, D.; Wang, S. RSC Adv. 2018, 8, 28414.

[45] Wang, Y.; Bao, L.; Liu, Z. H.; Pang, D. W. Anal. Chem. 2011, 83, 8130 .

[46] Zhu, H.; Ding, Y.; Wang, A.; Sun, X.; Wu, X. C.; Zhu, J. J. J. Mater. Chem. B 2015, 3, 458.

[47] Liu, X. Y.; Ren, J.; Su, L. H.; Gao, X.; Tang, Y. W.; Ma, T.; Zhu, L. J.; Li, J. R. Biosens. Bioelectron. 2017, 87, 203.

[48] (a) Holmes, P.; James, K. A. F.; Levy, L. S. Sci. Total Environ. 2009 408, 171; (b)Tchounwou, P. B.; Ayensu, W. K.; Ninashvili, N.; Sutton, D. Environ. Toxicol. 2003, 18, 149.

[49] (a) Yang, Y. B.; Yang, X. D.; Yang, Y. J.; Yuan, Q. Carbon 2018, 129, 380; (b) Wang, Q.; Chen, L.; Long, Y. T.; Tian, H.; Wu, J. C. Theranostics 2013, 3, 395.

[50] Wang, J.; Wei, T.; Li, X. Y.; Zhang, B. H.; Wang, J. X.; Huang, C.; Yuan, Q. Angew. Chem. Int. Ed. 2014, 53, 1616.

[51] Zhao, J.; Gao, J.; Xue, W.; Di, Z.; Xing, H.; Lu, Y.; Li, L. L. J. Am. Chem. Soc. 2018, 140, 578.

[52] Wu, S.; Duan, N.; Ma, X.; Xia, Y.; Wang, H.; Wang, Z.; Zhang, Q. Anal. Chem. 2012, 84, 6263.

[53] (a) Liu, Y.; Ouyang, Q.; Li, H.; Chen, M.; Zhang, Z.; Chen, Q. S. J. Agric. Food Chem. 2018, 66, 6188; (b) Wu, S. J.; Duan, N.; Shi, Z.; Fang, C. C.; Wang, Z. P. Talanta 2014, 128, 327.

[54] Xu, Y. X.; Meng, X. F.; Liu, J. L.; Dang, S.; Shi, L. Y.; Sun, L. N. CrystEngComm 2016, 18, 4032.

[55] Guan, X. L.; Li, Z. F.; Wang, L.; Liu, M. N.; Wang, K. L.; Yang, X. Q.; Li, Y. L.; Hu, L. L.; Zhao, X. L.; Lai, S. J.; Lei, Z. Q. Acta Chim. Sinica 2019, 77, 1268 (in Chinese). (关晓琳, 李志飞, 王林, 刘美 娜, 王凯龙, 杨学琴, 李亚丽, 胡丽丽, 赵小龙, 来守军, 雷自强, 化学学报, 2019, 77, 1268.) 